\title{
Review
}

Li Han*

\section{Research progress of siRNA in anti-influenza viral infection}

DOI: $10.2478 / \mathrm{ii}-2018-0019$

Received April 25, 2018; accepted May 04, 2018; published online June 29, 2018

\begin{abstract}
The harms of seasonal flu and global pandemic influenza have generally attracted attention. However, the currently administered influenza drugs and flu vaccines have certain limitations. Since the discovery of the small interfering RNA (siRNA) and its mediated RNA interference process, this molecule has been widely used in the study of anti-influenza viral infections because of its high specificity and strong selectivity. The results provided new concepts for the prevention and treatment of influenza virus. However, the siRNA still faces an enormous challenge despite extensive studies on this molecule. The research progress of siRNA in anti-influenza viral infection was reviewed in this study.
\end{abstract}

Keywords: siRNA, RNA interfering, influenza virus

Influenza virus is an orthomyxoviridae, enveloped, single-stranded, and negative-stranded RNA virus. This virus can be divided into A, B, and C types according to the matrix protein $\mathrm{M}$ and nuclear protein NP. Influenza $A$ is prone to antigenic drift and antigenic shift and is the main pathogen for seasonal flu and global pandemic influenza [1-3]. According to the statistics of World Health Organization, influenza can cause 3-5 million serious cases and 250,000 to 500,000 deaths annually worldwide [4].

At present, the commercially approved influenza drugs include M2 channel blockers (amantadine and rimantadine) and NA activity inhibitors (oseltamivir and zanamivir). However, strains resistant to these drugs have already emerged and cannot effectively respond to influenza pandemics $[5,6]$. Inoculation of influenza vaccine is an effective approach to prevent influenza, with the protection rate of $70 \%-80 \%$ [7]. However, given the rapid variation in the influenza virus, existing vaccines cannot induce cross-protective antibodies against new epidemic strains. Therefore, the influenza vaccine should be updated yearly according to the World Health Organization communiqué. Moreover, a large amount of vaccine is difficult to provide in a short period of time during an influenza pandemic [3,8]. The small interfering RNA (siRNA) has high specificity, strong selectivity, and high silencing efficiency and has recently become a hot spot for research on anti-influenza viral infection [9]. This paper reviews the progress and existing problems of siRNA in antiinfluenza viral infection.

\section{1 siRNA overview}

The siRNA is a short non-coding RNA molecule of approximately $21-25 \mathrm{nt}$ in length. This molecule is formed by the excises of exogenous double-strand RNA (dsRNA) through intracellular Dicer enzyme cleavage and can specifically silence targeted gene to inhibit the replication of the virus [10,11]. Pre-synthesized siRNA and short-hair RNA (shRNA) are two main types of siRNA. The former enters the cell by transient transfection and functions for approximately $48 \mathrm{~h}$. The shRNA is integrated into the cell genome through the vector and transcribed to form the shRNA, which can function stably for a long time [10]. The siRNA degradation of

Department of Ophthalmology, Yidu Central Hospital Affiliated to Weifang Medical College, Qingzhou, China

*Correspondence: Li Han, E-mail: hanliqz@163.com 
homologous mRNAs and silencing of target genes are referred to as RNA interference (RNAi) [12,13]. The RNAi process can be divided into two steps. In the first step, dsRNA is shear processed into 21-24 nt siRNA doublestrand molecule by double-strand-specific ribonuclease III Dicer [14]. In this process, the Dicer enzyme is first combined with TAR-RNA binding protein 2 (TRBP, also known as TARBP2) or protein-protected R (activated area conservation trust, PACT) to form a complex (Dicer-TRBP or Dicer-PACT), cutting dsRNA to form siRNA. Then, the siRNA antisense strand is combined with the RNA-induced silencing complex (RISC) [6,15]. The RISC consists of Ago-2, Dicer, TRBP, or PACT and is a key substance that degrades the target gene. In the second step, the siRNA combined with the RISC complex finds its target mRNA sequence through base complementation and under the action of the Ago-2 protein to degrade mRNA. This process blocks the translation of the protein. The Ago-2 protein is an RNA-induced, $\mathrm{Mg}^{2+}$-dependent, and RNase $\mathrm{H}$-activating molecule in the RNAi process. The endonuclease activity of the $3^{\prime}$-end PIWI region can effectively eliminate the phosphodiester bond of the target RNA and degrade target genes [16]. Given the high specificity and selectivity of siRNA, the siRNA-mediated RNAi process is widely used to prevent and control various viral infections.

\section{Study of siRNA in anti-influenza viral infection}

In 2003, Ge et al. [17] first applied the siRNA-mediated RNAi process to investigate the influenza viral infection. This study opened up a new approach for the treatment of influenza viral infection.

\subsection{Study of siRNA in anti-influenza viral infection}

The siRNA can specifically silence the influenza virus mRNA and inhibit the replication of the virus at different levels. Ge et al. [17] observed the effect of siRNA on H1N1 virus suppression in MDCK cells. The results showed that specific siRNA could inhibit the expression of approximately 50\% of influenza virus mRNA, while the effects on complementary RNA (cRNA) and virion RNA (vRNA) were not obvious. Moreover, the RNAi process did not affect the cell signaling pathway. The plaque test has shown that siRNA could reduce the influenza virus titer to 1/200, and three siRNAs (NP-1496, PA-2087, and PB1-2257) could reduce the titers of $\mathrm{A} / \mathrm{PR} / 8 / 34$ and $\mathrm{A} / \mathrm{WSN} / 33 \mathrm{H} 1 \mathrm{~N} 1$ viruses and even completely failed to detect the hemagglutination activity. Jain et al. [18] extended the length of the designed 19-nt siRNA to $27 \mathrm{nt}$. The results showed that increasing the length of siRNA inhibited the H1N1 and H3N2 multiple strains by more than $60 \%$, and the inhibitory effect was most obvious at $48 \mathrm{~h}$. In 2016, McMillen et al. [19] reported that although the NS1 gene-specific siRNAs downregulated the mRNA, the mRNA and protein levels of cell lysates NA and M were upregulated. However, the plaque test showed that the influenza virus titer decreased. Thus, defective influenza virus may have been produced during the RNAi process. Previous studies [20-22] constructed shRNAs into vectors, making their gene silencing more effective than that of siRNAs. However, their inhibitory effect was less pronounced than that of siRNAs, and their role was more pronounced in A549 cells than in MDCK cells. Hence, the siRNA in the A549 cells is more likely to escape degradation of lysosomes.

\subsection{Study of siRNA in anti-influenza viruses in chick embryos and animals}

The siRNA can inhibit the proliferation of influenza virus in chick embryos. Based on cell experiments, Ge et al. [17] first used liposomes to transfect siRNA into chick embryos. The siRNA was found to reduce virus titer to $1 / 200$ when the viral titers in the allantoic fluid were determined. The inhibitory effect was consistent with the cellular assay, and the siRNA without inhibitory effect on the MDCK cells also had no inhibitory effect on the chicken embryos. Cheng et al. [23] showed that the inhibitory effect of siRNA in the chick embryos was consistent with the detection results in the A549 cells. Given the large variability among chick embryos, the rapid growth of virus strains in the chick embryos, and the weaker inhibitory effects of siRNA, the use of chick embryos to study siRNA showed less effect on influenza viruses. 
The siRNA can inhibit the proliferation of influenza virus in animals and increase the protection rate of animals. Tompkins et al. [24] first intravenously injected siRNA to mice, and the virus titer was reduced after H1N1 challenge. Then, after 18 days of post-single injection of siRNA (NP-1 496 or PA-2 087) and two siRNAs to the mice, the protection rate of a single siRNA was $80 \%-90 \%$, and the two siRNA-treated mice survived, with $100 \%$ protection rate. The result demonstrated for the first time that siRNA could inhibit the proliferation of influenza virus in vivo, which was consistent with the results of Ge et al. [25]. In addition, Tompkins et al. [24] also found that after the siRNA was designed for H1N1infected mice with $\mathrm{H} 7 \mathrm{~N} 7$ and H5N1 subtypes, the survival rate of the mice was still more than 50\%. Subsequently, Rajput et al. [26] injected the siRNA into the nasal cavity and intravenously into the mice. The results indicated that mRNA and protein were inhibited by more than $90 \%$ and the inflammatory factors were also significantly reduced. Wei and Liu [6] injected the NP-shRNA-lentiviral vector into mice to reduce the virus titer of the lung tissue to $1 / 100$, and shRNA administered in the nasal cavity was more effective than intravenous injection. Animal experiments further confirmed that siRNA has an inhibitory effect on influenza virus.

\section{Study of anti-influenza viral infection after coupling of siRNA with other small molecules}

The siRNA can specifically silence mRNA, inhibit the proliferation of influenza virus, and increase the protection rate of animals. However, as a negatively charged exogenous nucleic acid molecule, siRNA is difficult to enter into cells and can be easily degraded within cells with a short-acting time. These characteristics seriously hinder the application of siRNA to influenza viral infection. In the past decade, studies of siRNA in anti-influenza viral infection have focused on the optimization of siRNAs [21,22,27-29]. These molecules have mainly been used in conjunction with other pro-cell types or synergistic nucleic acid molecules.

\subsection{Pro-cell carrier coupling}

Coupling with a pro-cell carrier can increase the efficiency of siRNA delivery in cells, uptake efficiency of siRNA into cells, and inhibition ratio of siRNA against H1N1 virus. Timin et al. [27] constructed siRNApolyelectrolyte carriers and outsourced biocompatible silica shells to form a spherical capsule with a diameter of approximately $100 \mathrm{~nm}$. After transfection of A549 and MDCK cells for $24 \mathrm{~h}$, the release rate of siRNA in the cells was $98 \%$, which was significantly higher than that of siRNA alone (only $1 \%$ ). With prolonged incubation time, the number of siRNAs that entered the cells was gradually increased, and further detection showed that the inhibitory effect on the H1N1 virus could be extended to $72 \mathrm{~h}$. Liang et al. [21] coupled siRNAs to $\mathrm{pH}$-modulating peptides to improve the efficiency of siRNA delivery to cells by changing the $\mathrm{pH}$. The results showed that the $\mathrm{pH}$-regulating peptide/siRNA complex reduced influenza virus titer in MDCK cells to 1/10. The virus titer in A549 cells was reduced to 1/9,000. After preparing this complex into a dry powder, the virus titer in A549 cells could still be reduced to 1/10,000-1/3,000. This result suggests that the pH-regulating peptide/siRNA complex could significantly enhance the efficiency of siRNA delivery and thus increase the inhibition rate.

\subsection{Nucleic acid molecule coupling}

Coupling of siRNAs with nucleic acid molecules with different functions can increase endogenousness and improve silencing efficiency and inhibition rate. Kumar et al. [28] linked the siRNA of PR8-M1 to a ribozyme-catalyzed sequence with a degradative nucleic acid to form an siRNA-ribozyme chimera to further increase the ability of siRNA to degrade nucleic acids. Consequently, the optimized siRNA-targeted 
silencing efficiency was increased by fourfold. Joshi et al. [22] found that the inhibition rate of influenza virus reached $80 \%$ after a sequence with an immunostimulatory function (5'-UGUGU-3') was added to the $5^{\prime}$-end of the NP-siRNA. This rate represented a fourfold increase compared with single siRNA inhibition group. Xu et al. [29] constructed the NP-1496 siRNA into a vector containing endogenous microRNA (miRNA) (forming shRNAmir-NP) to increase its endogenousness. After infection with PR8 live virus strain, NP protein was completely inhibited and the virus titer was reduced to 1/100. After 10 times increase in viral load, the shRNAmir-NP more significantly inhibited the NP protein and virus titers. Subsequently, PB1-2257 and NP-1496 siRNAs were constructed together into vectors. The protein expression of NP and PB1 was downregulated. However, the virus titer still dropped to $1 / 100$ probably because of the saturation of RISC in the RNAi process.

\section{Problems and prospects of siRNA in anti-influenza viral infection}

The siRNA has a high specificity, strong selectivity, silencing efficiency of $70 \%-75 \%$, and other beneficial characteristics. Hence, this molecule has been used in the study of anti-influenza viruses [30]. However, siRNAs have several problems in anti-influenza viral infections. Thus, the current research is restricted to cells, chick embryos, and animals and has not yet entered the clinical research stage. These problems are mainly in the following aspects. (1) The siRNA has an off-target effect. The siRNA silences the influenza virus target gene by base pairing with each other. When the host cell and target genes have similarities, the miRNA-like structural complexes formed in the RNAi process non-specifically interfere with the 3'-UTR of the host gene. This process silences the gene and even affects the pathways that the host miRNA regulates in the cells [31]. (2) The siRNAs are nucleic acids with numerous negatively charged molecules on their surfaces, and the number of phospholipid bilayers that actually penetrate the cell membrane is only $1 \%$, which reduces the inhibitory rate of siRNA on influenza [31]. (3) The siRNAs, as exogenous substances, are identified by a pattern recognition receptor to cause the body's innate immune response to enter the humoral circulation. These siRNAs are easily degraded in the liver and kidney and recognized and degraded by nucleases in the body. The effect of the siRNA synthesized externally in vivo can only be maintained for approximately $48 \mathrm{~h}$ [30-32]. These results make the siRNA inhibitory rate on the target gene unstable. Although the shRNA constructed on the vector can stably exhibit inhibitory effects in vivo, its effect is less than that of siRNA, and shRNA is difficult to prepare [30].

Given the defects of siRNAs during application, studies have increasingly focused on the modification of siRNAs. The design of siRNA is limited to the target ORF region, and predesigned siRNA is compared with host genes to reduce nonspecific responses [33]. Thus, target removal effects are reduced. Barrangou et al. [30] proposed to perform 2'-F and 2'-O- methyl modification on siRNA. Mimicking the natural RNA molecule can reduce the off-target effect and the innate immune response. Moreover, coupling siRNAs with various pro-cell carriers to form small molecules can increase the permeability of cells and reduce innate immunity responses [27,31]. In addition, when siRNA is applied to the stem-loop structure of mRNA, the siRNA inhibition rate can be increased [34]. The siRNA designed for $\mathrm{M}$ and NS transcripts with primary transcripts exhibited enhanced silencing effect [19]. Mutating $3^{\prime}$ - of the siRNA with one base did not affect siRNA heterosexual recognition and exerted its silencing function. The siRNA silencing efficiency can be improved for the activity of the target gene of siRNA and siRNA chemical modification [33].

Although these measures have improved the inhibitory effect of siRNA to a certain extent, each approach has its own advantages and disadvantages. Therefore, various factors should be fully considered when designing siRNAs. With the step-by-step solution of these issues, siRNA will provide new ideas for anti-influenza viral infection.

Acknowledgments: None.

Conflict of interest: The author states no conflicts of interest. 


\section{References}

[1] Coates D.M., Sweet C., Quarles J.M., Overton H.A., Smith H., Severity of fever in influenza: studies on the relation between viral surface antigens, pyrexia, level of nasal virus and inflammatory response in the ferret, J. Gen. Virol., 1985, 66(Pt 7), 1627-1631.

[2] Fang H., Yuan L., Li C., Pandemic influenza and type A H1N1 influenza, Chin. Pharm. Affairs, 2009, 23(12), 1216-1220.

[3] Li C., Review on who risk assessment and strategic action against pandemic influenza, Chin. Pharm.Affairs, 2006, 20(6), 370-374.

[4] WHO. Live Report on Flu (Seasonal). (2016-11) [2017-03-20], http://www.Who.int/mediacentre/factsheets/fs211/zh.

[5] Wang L., Li K., Advances in outbreak of influenza A pandemic caused by a novel type H1N1 in 2009, Prog. Microbiol. Immunol., 2012, 40(3), 79-82.

[6] Wei X., Liu W., Inhibitory effects of oseltamivir, zanamivir, and peramivir on the human parainfluenza neuraminidase of the H7N9 influenza virus, J. Zhengzhou Univ. (Med. Ed.), 2014, 49(3), 433-435.

[7] Thomas M., Ge Q., Lu J.J., Klibanov A.M., Chen J., Polycation-mediated delivery of siRNAs for prophylaxis and treatment of influenza virus infection, Expert Opin. Biol. Ther., 2005, 5(4), 495-505.

[8] Li C., Wang Z., Issues in developing the pandemic influenza vaccine and related regulatory activities, Chin. Pharm. Affairs, 2007, 21(8), 563-568.

[9] Fire A., Xu S., Montgomery M.K., Kostas S.A., Driver S.E., Mello C.C., Potent and specific genetic interference by doublestranded RNA in Caenorhabditis elegans, Nature, 1998, 391(6669), 806-811.

[10] Azlan A., Dzaki N., Azzam G., Argonaute: the executor of small RNA function, J. Genet. Genomics, 2016, 43(8), $481-494$.

[11] Su L., Zhang P., Wangyang J., Zhong R.,, Progress on the inhibition of hepatitis B virus by siRNA strategy, China Biotechnol., 2014, 34(9), 102-107.

[12] Elbashir S.M., Harborth J., Lendeckel W., Yalcin A., Weber K., Tuschl T., Duplexes of 21-nucleotide RNAs mediate RNA interference in cultured mammalian cells, Nature, 2001, 411(6836), 494-498.

[13] Hammond S.M., Caudy A.A., Hannon G.J., Post-transcriptional gene silencing by double-stranded RNA, Nat. Rev. Genet., 2001, 2(2), 110-119.

[14] CsoRba T., Pantaleo V., Burgy N.J., RNA silencing: an antiviral mechanism, In: L. Gad, P.C. John (eds.), Advances in virus research, Vol. 75, Chap. 2, Academic Press, Manhattan, NY, 2009. 230.

[15] O'Keefe EP. siRNA and shRNA: Tools for inhibiting protein expression through gene silencing. (2013-06-05) [2017-03-20], http://www.labome.cn/method/siRNAs-and-shRNAs-Tools-for-Protein-Knockdown-by-Gene-Silencing.html.

[16] Ge Q., Xu J.J., Evans D.M., Mixson A.J., Yang H.Y., Lu P.Y., Leveraging therapeutic potential of multi-targeted siRNA inhibitors, Future Med. Chem., 2009, 1(9), 1671-1681.

[17] Ge Q., McManus M.T., Nguyen T., Shen C.H., Sharp P.A., Eisen H.N., et al., RNA interference of influenza virus production by directly targeting mRNA for degradation and indirectly inhibiting all viral RNA transcription, Proc. Natl. Acad. Sci. U.S.A., 2003, 100(5), 2718-2723.

[18] Jain B., Jain A., Prakash O., Singh A.K., Dangi T., Singh M., et al., In vitro validation of self designed "universal human influenza A siRNA, Indian J. Exp. Biol., 2015, 53(8), 514-521.

[19] McMillen C.M., Beezhold D.H., Blachere F.M., Othumpangat S., Kashon M.L., Noti J.D., Inhibition of influenza A virus matrix and nonstructural gene expression using RNA interference, Virology, 2016, 497, 171-184.

[20] Stoppani E., Bassi I., Dotti S., Lizier M., Ferrari M., Lucchini F., Expression of a single siRNA against a conserved region of NP gene strongly inhibits in vitro replication of different Influenza A virus strains of avian and swine origin, Antiviral Res., 2015, 120, 16-22.

[21] Liang W., Chow M.Y., Lau P.N., Zhou Q.T., Kwok P.C., Leung G.P., et al., Inhalable dry powder formulations of siRNA and pH-responsive peptides with antiviral activity against H1N1 influenza virus, Mol. Pharm., 2015, 12(3), 910-921.

[22] Joshi G., Dash P.K., Agarwal A., Sharma S., Parida M., Bifunctional siRNA containing immunostimulatory motif enhances protection, Curr. Gene Ther., 2015, 15(5), 492-502.

[23] Cheng C., Yao L., Chen A., Jia R., Huan L., Guo J., et al., Inhibitory effect of small interfering RNA specific for a novel candidate target in PB1 gene of influenza A virus, J. Drug Target., 2009, 17(2), 133-139.

[24] Tompkins S.M., Lo C.Y., Tumpey T.M., Epstein S.L., Protection against lethal influenza virus challenge by RNA interference in vivo, Proc. Natl. Acad. Sci. U.S.A., 2004, 101(23), 8682-8686.

[25] Ge Q., Filip L., Bai A., Nguyen T., Eisen H.N., Chen J., Inhibition of influenza virus production in virus-infected mice by RNA interference, Proc. Natl. Acad. Sci. U.S.A., 2004, 101(23), 8676-8681.

[26] Rajput R., Khanna M., Kumar P., Kumar B., Sharma S., Gupta N., et al., Small interfering RNA targeting the nonstructural gene 1 transcript inhibits influenza A virus replication in experimental mice, Nucleic Acid Ther., 2012, 22(6), 414-422.

[27] Timin A.S., Muslimov A.R., Petrova A.V., Lepik K.V., Okilova M.V., Vasin A.V., et al., Hybrid inorganic-organic capsules for efficient intracellular delivery of novel siRNAs against influenza A (H1N1) virus infection, Sci. Rep., 2017, 7(1), 102.

[28] Kumar P., Sood V., Vyas R., Gupta N., Banerjea A.C., Khanna M., Potent inhibition of influenza virus replication with novel siRNA-chimeric-ribozyme constructs, Antiviral Res., 2010, 87(2), 204-212.

[29] Xu F., Liu G., Liu Q., Zhou Y., RNA interference of influenza A virus replication by microRNA-adapted lentiviral loop short hairpin RNA, J. Gen. Virol., 2015, 96(10), 2971-2981. 
[30] Barrangou R., Birmingham A., Wiemann S., Beijersbergen R.L., Hornung V., Smith Av, Advances in CRISPR-Cas9 genome engineering: lessons learned from RNA interference, Nucleic Acids Res., 2015, 43(7), 3407-3419.

[31] Dowdy S.F., Overcoming cellular barriers for RNA therapeutics, Nat. Biotechnol., 2017, 35(3), 222-229.

[32] BoettcheR M., Mcmanus M.T., Choosing the right tool for the job: RNAi, TALEN, or CRISPR, Mol. Cell, 2015, 58(4), 575-585.

[33] Fakhr E., Zare F., Teimoori-Toolabi L., Precise and efficient siRNA design: a key point in competent gene silencing, Cancer Gene Ther., 2016, 23(4), 73-82.

[34] Behera P., Nagarajan S., Murugkar H.V., Kalaiyarasu S., Prakash A., Gothalwal R., et al., siRNAs targeting PB2 and NP genes potentially inhibit replication of highly pathogenic H5N1 avian influenza virus, J. Biosci., 2015, 40(2), 233-240. 